Research Paper

\title{
A Novel Semisynthetic Molecule Icaritin Stimulates Osteogenic Differentiation and Inhibits Adipogenesis of Mesenchymal Stem Cells
}

Hui Sheng ${ }^{1,2,3^{*} \bowtie}$, Xue-fei Rui ${ }^{12^{*}}$, Chun-Jun Sheng ${ }^{1}$, Wen-Jun Li ${ }^{1}$, Xiao-Yun Cheng ${ }^{1}$, Navina Priya Jhummon ${ }^{1}$, Yong-Chun $\mathrm{Yu}^{1}$, Shen $\mathrm{Qu}^{1}$, Ge Zhang ${ }^{4}$, Ling Qin ${ }^{4}$

1. Department of Endocrinology and Metabolism, Shanghai Tenth People's Hospital, Tongji University School of Medicine, Shanghai, China;

2. Department of Clinical Medicine, Nanjing Medical University, Nanjing, China;

3. Advanced Institute of Translational Medicine, Tongji University, Shanghai, China;

4. Department of Orthopaedics \& Traumatology, The Chinese University of Hong Kong, Hong Kong SAR, China.

${ }^{*}$ Co-first author

$\triangle$ Corresponding author: Dr. Hui Sheng, Department of Endocrinology and Metabolism, Shanghai Tenth People's Hospital, Tongji University School of Medicine, Shanghai, China, e-mail: shenghui@tongii.edu.cn. Dr. Ling Qin, Professor and Director of Musculoskeletal Research Laboratory, Department of Orthopaedics and Traumatology, Prince of Wales Hospital, The Chinese University of Hong Kong, Shatin, N.T., Hong Kong, e-mail: lingqin@cuhk.edu.hk.

( ) Ivyspring International Publisher. This is an open-access article distributed under the terms of the Creative Commons License (http://creativecommons.org/ licenses/by-nc-nd/3.0/). Reproduction is permitted for personal, noncommercial use, provided that the article is in whole, unmodified, and properly cited.

Received: 2013.02.17; Accepted: 2013.04.10; Published: 2013.04.23

\begin{abstract}
Background We previously reported that the constitutional flavonoid glycosides derived from herb Epimedium (EF, composed of seven flavonoid compounds with common nuclear stem) exerted beneficial effects on the bone, including promoting bone formation and inhibiting bone marrow fat deposition. Recent in vivo study showed that Icaritin was a common metabolite of these constitutional flavonoid glycosides, indicating that Icaritin is a bioactive compound. The present study was designed to investigate whether Icaritin could promote osteogenic differentiation and suppress adipogenic differentiation of marrow mesenchymal stem cells (MSCs).

Methods Primary MSCs were harvested from adult mice and exposed to Icaritin to evaluate whether it could promote osteogenesis and suppress adipogenesis using the following assays: determination of alkaline phosphatase (ALP) activity and mineralization; mRNA expression of osteogenic differentiation marker Runx2; osteocalcin and bone sialoprotein (BSP) by RT-PCR; quantification of adipocyte-like cells by Oil Red $\mathrm{O}$ staining assay and mRNA expression for adipogenic differentiation markers peroxisome proliferator-activated receptor gamma (PPARY); adipocyte fatty acid binding protein (aP2) and lipoprotein lipase (LPL) by RT-PCR. For the underlying mechanism, glycogen synthase kinase-3beta (GSK3 $\beta$ ) and $\beta$-catenin were also explored by western blotting.

Results Icaritin promoted osteogenic differentiation and maturation of MSCs as indicated by increased mRNA expression for Runx2, osteocalcin and BSP, and enhanced ALP activity and mineralization; Icaritin inhibited adipogenic differentiation, as indicated by decreased mRNA expression for PPARY, LPL, aP2, and suppressed formation of adipocyte-like cells; Icaritin inactivated GSK3 $\beta$ and suppressed PPARY expression when promoting osteogenesis and suppressing adipogenesis of MSCs.
\end{abstract}


Conclusion This was the first study demonstrating that the novel semisynthetic molecule Icaritin could stimulate osteogenic differentiation and inhibit adipogenesis of MSCs, which was associated with the suppression of GSK3 $\beta$ and PPARY.

Key words: Mesenchymal stem cells, Icaritin, Adipogenesis, Osteogenesis, GSK3 $\beta$, Osteoporosis.

\section{Introduction}

Many Chinese-herb-derived formulas have shown their protective effects on bone in experimental and clinical studies, but the bioactive component(s) in these formulas are difficult to identify. This has greatly hampered the development and globalization of herbal medicine. We have previously reported the preclinical and clinical findings of constitutional flavonoid glycosides derived from herbal Epimedium (EF, composed of seven flavonoid compounds with common nuclear stem) to treat postmenopausal osteoporosis and steroid-associated bone disorders. EF exerted beneficial effects on bone, including promotion of bone formation and inhibition of bone marrow fat deposition (1-3). However, the bioactive compound and the underlying mechanisms remain unclear.

Decreased bone formation and increased bone marrow fat deposition are common feature in postmenopausal osteoporosis and other bone disorders $(4,5)$. These have been explained by suppressed osteogenesis and enhanced adipogenesis of marrow mesenchymal stem cells (MSCs) (6-9). Wang et al. and Mukherjee et al. reported that treating osteoporotic animals with bioactive agents that enhanced osteogenic differentiation of MSCs, also promoted bone formation $(10,11)$. These results suggested that the beneficial effect of our efficacy-proven EF on bone might be due to the modulation of the balance between osteogenic and adipogenic differentiation of MSCs by its bioactive compound(s). Recent in vivo study showed that Icaritin was the common metabolic compound of the seven flavonoid compounds in this formula (12), indicating that Icaritin was a relevant bioactive compound. The present study aimed to investigate whether Icaritin could stimulate osteogenic and inhibit adipogenic differentiation of MSCs.

\section{Materials and Methods}

\section{Reagents}

All reagents were of the highest purity. Icaritin was purchased from the National Institute of Control of Pharmaceuticals and Biological Products (Beijing, China). Cell culture reagents and PCR primers were purchased from Invitrogen (Basel, Switzerland). If not indicated otherwise, all other reagents were pur- chased from Sigma-Aldrich (St. Louis, MO, USA).

\section{MSCs isolation, expansion and culture}

Bone marrow was harvested from femora and tibiae of 3 4-month old wild-type C57BL/6N mice, and used for collecting MSCs according to a previously published protocol (13). The cell suspension was prepared in phosphate buffered saline (PBS), and then layered onto Lymphoprep ${ }^{\mathrm{TM}}(1.077 \mathrm{~g} / \mathrm{ml}$; Nycomed-Amersham, Norway) for density gradient centrifugation $(1840 \mathrm{rpm}, 30 \mathrm{mins}$, at room temperature). The mononuclear cells were collected from the interface of the Ficoll solution, and then resuspended in Dulbecco's Modified Eagle's medium (DMEM) that contained $10 \%$ fetal calf serum (FCS), $100 \mathrm{U} / \mathrm{ml}$ penicillin, $100 \mathrm{mg} / \mathrm{ml}$ streptomycin, $2.5 \mu \mathrm{g} / \mathrm{ml}$ fungizone and $2 \mathrm{mM}$ L-glutamine (Invitrogen, Paisley, UK), and then seeded into T75 flasks (Nunc, Roskilde, Denmark) at a density of $1-3 \times 10^{5}$ cells $/ \mathrm{cm}^{2}$. These cells were incubated at $37^{\circ} \mathrm{C}$ in humidified $5 \% \mathrm{CO}_{2}$. When the primary MSCs reached confluence, they were separated using Trypsin-EDTA solution and passed at $1 \times 10^{4}$ cells $/ \mathrm{cm}^{2}$ into T75 flasks. The subsequently obtained MSCs were used in the following experiments.

\section{Osteogenic stimulation}

MSCs were seeded at a density of 5000 cells $/ \mathrm{cm}^{2}$ in 6-well culture plate in DMEM. When MSCs reached confluence, the medium was replaced with the osteogenic differentiation medium (OM) whose composition was DMEM consisting of $10 \%$ FCS, $0.3 \mathrm{mM}$ ascorbic acid, $10 \mathrm{mM} \beta$-glycerophosphate and $10^{-8} \mathrm{M}$ dexamethasone (14). The treatment consisted of either vehicle $(0.1 \%$ dimethylsulfoxide) or increasing concentrations of Icaritin $(0.1-10 \mu \mathrm{M})$. The cells were harvested at different points in time to determine the alkaline phosphatase (ALP) activity and the degree of mineralization, and the determination of the mRNA expression for Runx2, BSP and osteocalcin.

\section{Adipogenic stimulation}

MSCs were seeded at a density of 5000 cells $/ \mathrm{cm}^{2}$ in the 6-well culture plate in DMEM. When MSCs reached confluence, the medium was changed to the adipogenic differentiation medium (AM) whose composition was DMEM consisting of $10 \%$ FCS, 
$0.5 \mu \mathrm{g} / \mathrm{ml}$ insulin, $10^{-8} \mathrm{M}$ dexamethasone and $0.5 \mathrm{mM}$ isobutylmethylxanthine (14). The treatment consisted of either vehicle $(0.1 \%$ dimethylsulfoxide) or increasing concentrations of Icaritin $(0.1-10 \mu \mathrm{M})$. Oil Red O staining for adipogenesis and mRNA expression for PPARY, LPL and aP2 were evaluated at the indicated points in time.

\section{Determination of Alkaline phosphatase (ALP) activity}

ALP activity was measured in MSCs using a previously described method (13). The cells were washed briefly with cold PBS, and then lysed with cold $\left(4^{\circ} \mathrm{C}\right)$ diethanolamine (DEA) buffer $(2 \mathrm{M} \mathrm{HCl}$, $0.01 \mathrm{M} \quad \mathrm{MgCl}_{2} .6 \mathrm{H}_{2} \mathrm{O}, 0.5 \%$ diethanolamine, $\mathrm{pH}$ 10.1-10.5). After adding 50 $\mathrm{pl}$ of 3mM 4-nitrophenyl phosphate hexahydrate (PNPP) substrate solution to each well, ALP activity was determined at room temperature using a microplate reader (Ultrospec 3000, Pharmacia Biotech, USA) at $405 \mathrm{~nm}$. The ALP activity was expressed as activity/cell protein which was measured using the BCA protein assay kit (Pierce, USA).

\section{Mineralization Assay}

Mineralization of MSCs was analyzed after 18 days' osteogenic induction. To this end, cells were fixed with $95 \%$ ethanol for 30 minutes, stained with $2 \%$ alizarin red for 20 minutes, and then washed with distilled water. The MetaMorph image Analysis Software (Universal Imaging Corporation ${ }^{\mathrm{TM}}$, Downingtown, PA, USA) was used to quantify the mineralized nodules, and the percentage of mineralized nodules was expressed as a fraction of the total measured area as previously described (15).

\section{Quantitative Real-time PCR}

Cells were collected at the indicated point in time during the osteogenic and adipogenic stimulation. The total RNA was extracted by Trizol reagent after washing them with PBS. The extract was reverse-transcripted under the following conditions: $42^{\circ} \mathrm{C}$ for 45 mins, and $99^{\circ} \mathrm{C}$ for 5 mins, and the cDNA product was stored at $-20^{\circ} \mathrm{C}$. Forward and reverse PCR primers were synthesized for Runx2, osteocalcin, BSP, PPARy, LPL, aP2, and GAPDH based on published paper $(16,17)$. The forward and reverse primers were: Runx2 (5'-AACCCACGGCCCTCCCTGAA CTCT-3' and 5'-ACTGGCGGGGTGTAGGTAAAGG TG-3'), osteocalcin (5'-ACCCTGGCTGCGCTCTGTC TCT-3' and 5'-GATGCGTTTGTAGGCGG-TCT TCA-3'), BSP( 5'-CAGAGGAGGCAAGCGTCACT-3' and 5'- CTGTCTGGGTGCCAACACTG-3'), PPARY (5'-GGGTCAGCTCTTGTGAATGG-3' and 5'-CTGA TGCACTGCCTATGAGC-3'), LPL (5'-AGGTCATCT TCTGTGCTAGG-3' and $5^{\prime}$-ATGCTGGAAGACCTG
CTATG-3') $\quad$ aP2 (5'-TGTGATGCCTTTGTG GGAACC-3' and 5'-CGTCTGCGGTGATTTCATC-3') and GAPDH (5'-CACCATGGAGAAGGCCGGGG-3' and 5'-GACGGACACATTGGGGGTAG-3). RT-PCR was performed and analyzed in a capillary real-time thermocycler (LightCycler, Roche Diagnostics, USA). Amplification was done in the presence of SYBR Green 1 . The PCRs were done in a $20 \mu \mathrm{l}$ reaction in which cDNA was mixed with LightCycler-Fastart DNA Master SYBR Green, and contained 10pmol of forward and reverse primer and $4 \mathrm{mM} \mathrm{MgCl}_{2}$ respectively. The samples were amplified in a thermocycler under the following conditions: 50 cycles at $95^{\circ} \mathrm{C}$ for 10 mins, 50 cycles at $95^{\circ} \mathrm{C}$ for 10 secs, 50 cycles at $55-60^{\circ} \mathrm{C}$ depending on the primer set for 10 secs, and 50 cycles at $72^{\circ} \mathrm{C}$ for 30 secs. PCR product accumulation was monitored at multiple points during each cycle by measuring the increase in fluorescence that was generated by the binding of SYBR Green 1 to dsDNA. Data analysis was performed with "Fit Point Method" in the LightCycler software 3.3 (Roche Diagnostics). Relative quantification was made against the expression levels of the house keeping gene GAPDH (18).

\section{Oil Red O Staining Assay}

The number of adipocyte-like cells was quantified on day 18 using the Oil Red O staining method (8). Briefly, the cells were fixed with $10 \%$ neutral buffered formalin, incubated with $60 \%$ propylene glycol, and then stained with $0.5 \%$ (wt/vol) Oil Red O in $60 \%$ propylene glycol for 30 minutes. After staining, the cells were rinsed thoroughly with distilled water, and then incubated for 1 hour at room temperature after adding $1 \mathrm{ml}$ of isopropyl alcohol. Aliquots of the extracted Oil Red O were measured at 510 $\mathrm{nm}$ with spectrophotometer (Ultrospec 3000, Pharmacia Biotech, USA). The values were normalized to cells protein which was measured using the BCA protein assay kit (Pierce, USA).

\section{Western Blot Analysis}

Whole cell proteins were extracted by protein extraction reagents kit (Pierce, USA). The protein content in each sample was measured using the BCA protein assay kit (Pierce, USA). Thirty micrograms of protein was separated by $10 \%$ SDS-PAGE and transferred to PVDF membrane by Trans-Blot SD Semi-Dry Electrophoretic Transfer Cell (Bio-Rad, USA). After blocking with 5\% non-fat milk for half an hour, the membrane was incubated overnight with primary antibodies against phospho-GSK3 $\beta$ (ser9, clone 2D3; Upstate., Lake Placid, NY), total-GSK3 $\beta$ (Chemicon, Billerica, MA), total $\beta$-catenin (BD, Bedford,MA), and $\beta$-actin (Santa Scuz Biotechnology, CA, USA), and 
then incubated with a secondary conjugated horseradish peroxidase antibody (Santa Cruz Biotechnology, CA, USA) for one hour after washing with TBS. The proteins were then visualized using a chemiluminescence detection kit (Santa Cruz Biotechnology, CA, USA) $(19,20)$.

\section{Statistical analysis}

Data are presented as mean \pm standard deviation (SD). The differences between the groups were analyzed by one-way ANOVA using SPSS software 13.0 (SPSS Inc., Chicago, IL, USA). Statistical significance was set at $P<0.05$.

\section{Results}

\section{Icaritin promotes osteogenic differentiation and maturation of MSCs}

To investigate the effects of Icaritin on osteogenic differentiation of MSCs, mRNA expression of Runx2 for the lineage-specific gene of osteogenic differentiation, BSP for osteogenesis protein, and osteocalcin for the mature osteoblast marker, together with the ALP activity and the extent of matrix mineralization were determined on day 3, 6 and 18 based on the preliminary experiments respectively.

\section{Genes expression profile}

As compared with MSCs in the osteogenic medium without Icaritin treatment (control group), the mRNA expression for Runx 2 was increased by 2-fold, 4.4-fold and 7.9-fold in $0.1 \mu \mathrm{M}, 1 \mu \mathrm{M}$ and $10 \mu \mathrm{M}$ Icaritin treatment groups respectively (Fig. 1A). The mRNA for BSP was increased by 1.5-fold, 1.9-fold and 3.4-fold in $0.1 \mu \mathrm{M}, 1 \mu \mathrm{M}$ and $10 \mu \mathrm{M}$ Icaritin treatment groups respectively (Fig. 1B). The mRNA for osteocalcin was increased by 1.1-fold, 2.2-fold and 3.3-fold in $0.1 \mu \mathrm{M}, 1 \mu \mathrm{M}$ and $10 \mu \mathrm{M}$ Icaritin treatment groups respectively (Fig. 1C).

\section{ALP activity}

As compared with MSCs in the osteogenic medium without Icaritin treatment, the ALP activity was enhanced by $50.4 \%, 90.9 \%$ and $154.5 \%$ in $0.1 \mu \mathrm{M}, 1 \mu \mathrm{M}$ and $10 \mu \mathrm{M}$ Icaritin treatment groups respectively (Fig. 1D). With 10uM Icaritin treatment, the peak ALP activity of MSCs was increased by $53.8 \%$ as compared to the control group. In addition, Icaritin prolonged the peak ALP expression period and there was a total of $160 \%$ increase in ALP activity within the first 12 days of treatment (Fig. 1E).

\section{Mineralization}

In the osteogenic medium, MSCs began to form mineralized nodules in two weeks, and matrix mineralization was quantified on day 18. Icaritin showed a dose-dependent effect on matrix mineralization. The mineralization area was increased by $50 \%, 130 \%$ and $220 \%$ in $0.1 \mu \mathrm{M}, 1 \mu \mathrm{M}$ and $10 \mu \mathrm{M}$ Icaritin groups respectively as compared to the control group (Fig. 1F, 1G).

\section{GSK $3 \beta$ is involved in the regulation of Icari- tin-induced osteogenic differentiation of MSCs}

The role of GSK3 $\beta$ in Icaritin-treated MSCs was evaluated. After Icaritin treatment, there was no significant change in total GSK3 $\beta$ expression, but the pGSK3 $\beta$ was increased by 1.2-fold, 4.1-fold and 11.3 -fold in $0.1 \mu \mathrm{M}, 1 \mu \mathrm{M}$ and $10 \mu \mathrm{M}$ Icaritin groups respectively as compared to the control group. As the target gene of GSK3 $\beta$, beta-catenin expression also showed a 1.1-fold, 7.3-fold and 14.1-fold increase in $0.1 \mu \mathrm{M}, 1 \mu \mathrm{M}$ and $10 \mu \mathrm{M}$ Icaritin groups respectively (Fig. 1H, 1I, 1J).

\section{Icaritin inhibits adipogenic differentiation of MSCs}

To investigate the effects of Icaritin on adipogenic differentiation of MSCs, the mRNA expression of PPARY for the lineage-specific transcription factor and $\mathrm{aP2}$ and LPL for the adipocyte-specific markers were determined by RT-PCR on day 3. The adipocyte-like cells were quantified using Oil Red O staining method based on the preliminary experiments.

\section{Genes expression profile}

As compared with MSCs in the control medium, the mRNA expression for PPARy, LPL and aP2 was increased by 9.3-fold, 7.1-fold and 4.2-fold in adipogenic medium. After adding Icaritin in the adipogenic medium, these genes expressions were significantly inhibited. The PPARY expression was inhibited by $39.8 \%, 66 \%$ and $79.6 \%$ in $0.1 \mu \mathrm{M}, 1 \mu \mathrm{M}$ and $10 \mu \mathrm{M}$ Icaritin groups respectively. The LPL expression was inhibited by $25 \%, 48.1$ and $65.4 \%$, and aP2 mRNA expression was inhibited by $32.7 \%, 51.9 \%$ and $67.3 \%$ in $0.1 \mu \mathrm{M}, 1 \mu \mathrm{M}$ and $10 \mu \mathrm{M}$ Icaritin groups respectively (Fig. 2A, 2B, 2C).

\section{Quantification of adipocyte-like cells}

Icaritin inhibited adipocyte-like cells formation in a dose-dependent manner. After two weeks of culture, it showed that there was much more adipocyte-like cells formation in adipogenic group as compared to the control group, and there were fewer adipocyte-like cells formation after the Icaritin treatment (Fig. 2D). The quantification data showed that Icaritin suppressed adipocyte-like cells formation in a dose-dependent manner, and the suppression rate was increased from $30 \%$ to $47 \%$ and $75 \%$ in $0.1 \mu \mathrm{M}$, $1 \mu \mathrm{M}$ and $10 \mu \mathrm{M}$ Icaritin treatment groups respectively (Fig. 2E). 


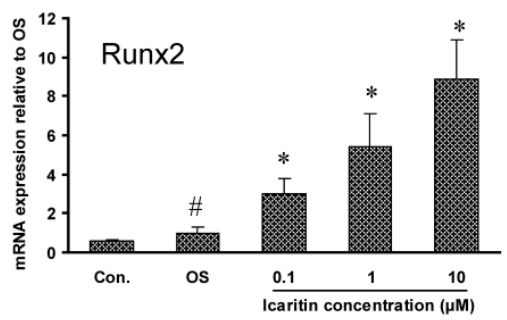

A

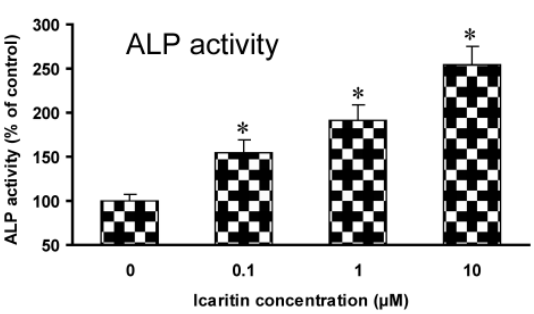

D

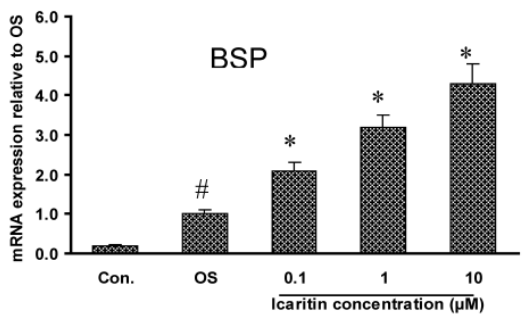

B

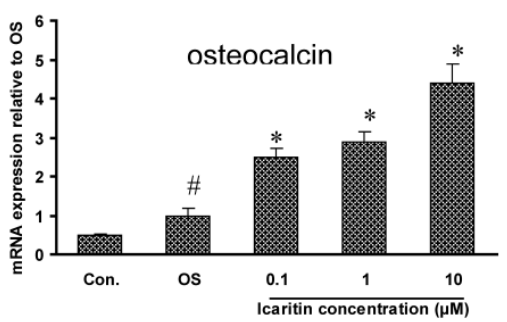

C

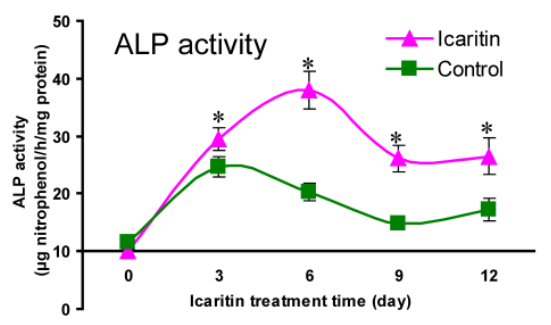

E

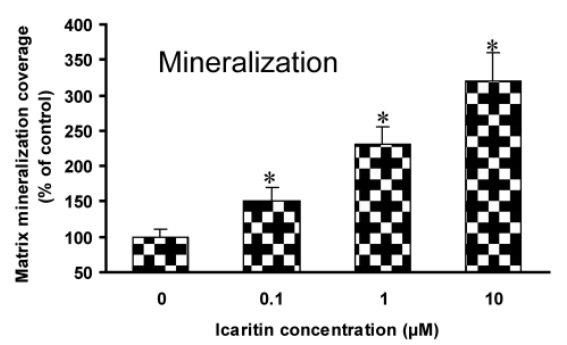

F

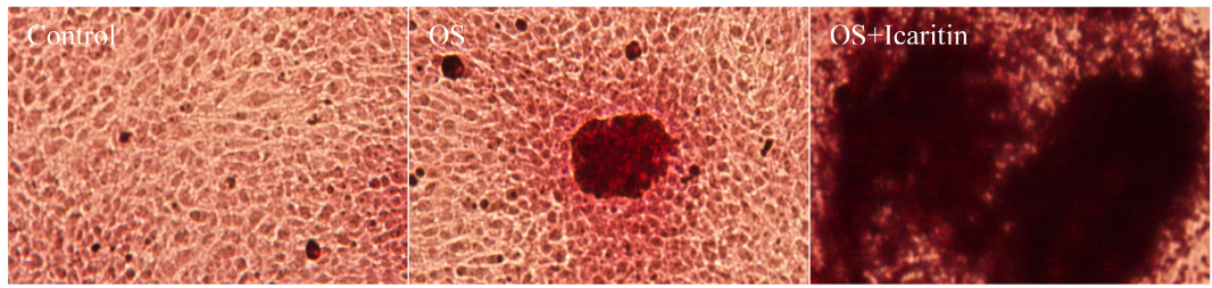

G

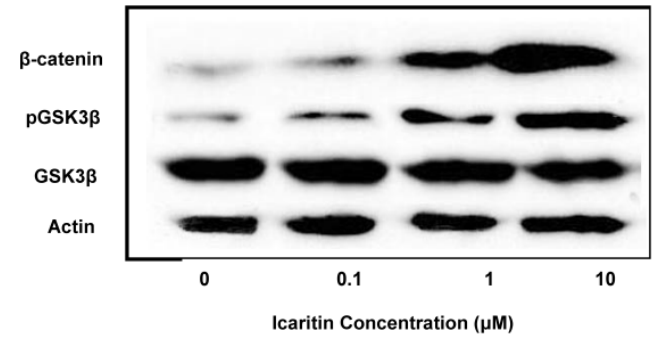

H

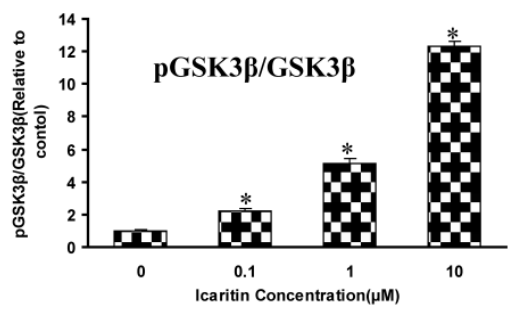

I

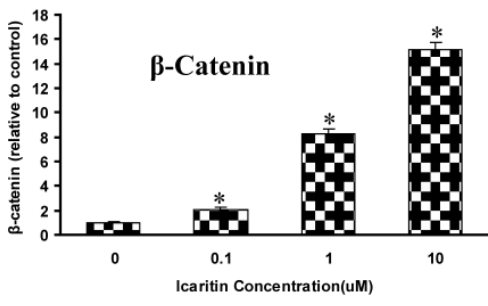

Fig. I The effects of Icaritin on osteogenic differentiation, maturation and mineralization of MSCs. A, B\&C: The effects of Icaritin on the mRNA expression for Runx2, BSP and osteocalcin in MSCs were compared between control group (Control), osteogenic medium(OS) and Icaritin treatment groups (OS plus with Icaritin). Results from a representative of three experiments are shown, reported as mean \pm SD of quadruplicate determinations $(* p<0.05$ vs. OS, \#P<0.05 vs Control ). D: The effects of different doses of Icaritin on ALP activity in MSCs. Results from a representative of three experiments are shown,

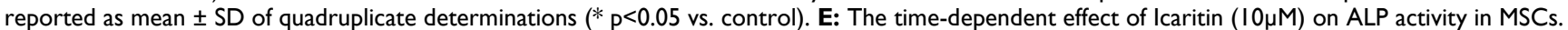
Results from a representative of three experiments are shown, reported as mean \pm SD of quadruplicate determinations $(* p<0.05 \mathrm{vs.} \mathrm{control} \mathrm{for} \mathrm{each} \mathrm{time}$ point). F: Quantification data showing the matrix mineralization area in control and different doses of Icaritin treatment groups. Results from a representative of three experiments are shown, reported as mean $\pm S D$ of quadruplicate determinations ( $* 0.05$ vs. osteogenic medium). G: Representative pictures showing the alizarin red staining mineralization nodules in control, osteogenic medium(OS), and $10 \mu M$ of Icaritin treatment group(OS+lcaritin). $\mathbf{H}$, I\&J: Western blot results showing the proteins expression of GSK3 $\beta$, pGSK3 $\beta$ and $\beta$-catenin after different doses of Icaritin treatment. Results from a representative of three experiments are shown, reported as mean \pm SD of quadruplicate determinations $(* p<0.05$ vs. control). 


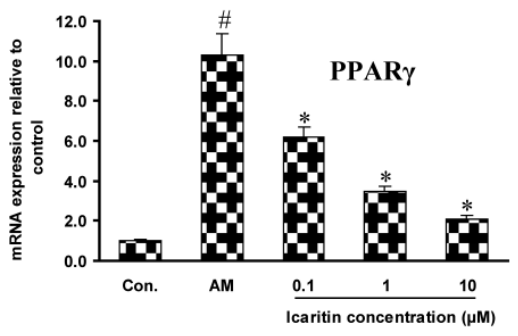

A

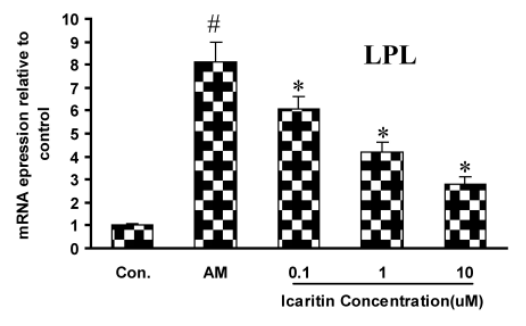

B

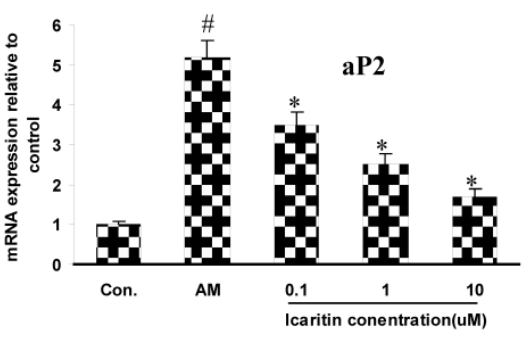

C

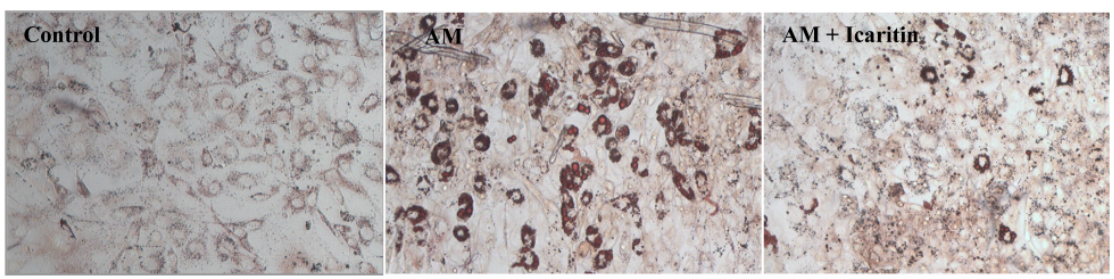

D

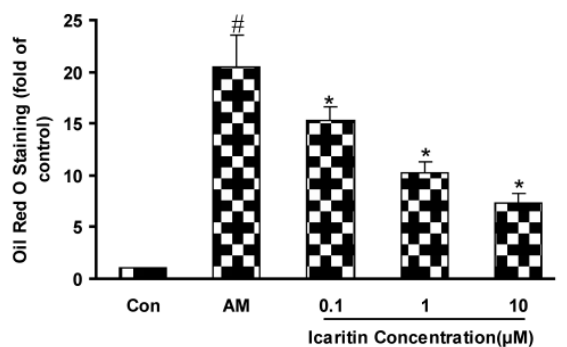

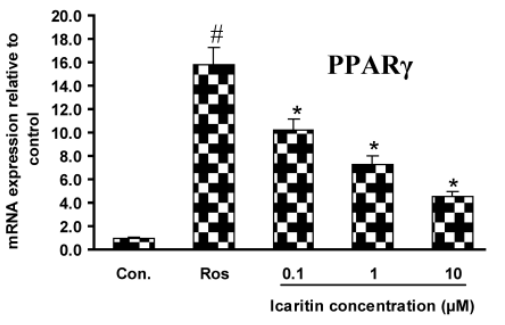

F

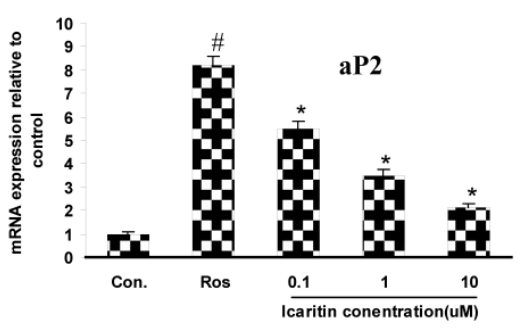

G
E

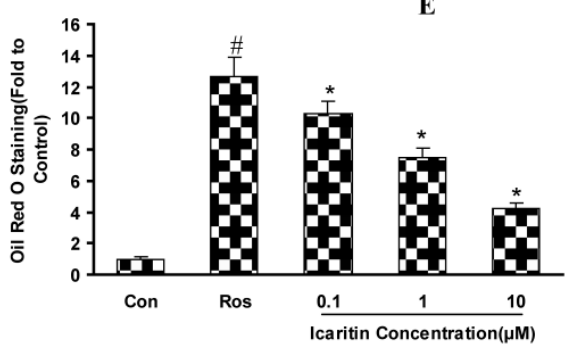

H

Fig. 2 The effects of Icaritin on adipogenic differentiation of MSCs. A,B\&C: RT-PCR results showing PPARy, LPL and aP2 mRNA expression in normal medium (control group), adipogenic medium (AM group) and adipogenic medium plus with lcaritin (Icaritin treatment group). Results from a representative of three experiments are shown, reported as mean \pm SD of quadruplicate determinations (\#p<0.05 vs. control group, *p<0.05 vs. AM group). D: Representative pictures showing the Oil-Red-O-positive adipocyte-like cells in control, AM, and I0 $\mu$ M of Icaritin treatment group. E: Quantification data showing the effects of Icaritin on adipocyte-like cells formation. Results from a representative of three experiments are shown, reported as mean \pm SD of quadruplicate determinations (\#p<0.05 vs. control group, ${ }^{*}<<0.05$ vs. AM group). F,G: RT-PCR results showing the PPARY and aP2 mRNA expression in normal medium (control group), rosiglitazone additive medium (Ros. $0.1 \mu M$ ) and rosiglitazone plus with Icaritin (Icaritin group). Results from a representative of three experiments are shown, reported as mean $\pm \mathrm{SD}$ of quadruplicate determinations (\#p<0.05 vs. control group, ${ }^{*} \mathrm{p}<0.05$ vs. Ros group). $\mathrm{H}$ : Oil Red $\mathrm{O}$ staining quantification showing the adipocyte-like cells formation in rosiglitazone and different doses of Icaritin treatment groups. Results from a representative of three experiments are shown, reported as mean \pm SD of quadruplicate determinations (\#p<0.05 vs. control group, *p<0.05 vs. Ros group).

\section{PPARY is involved in the regulation of Icari- tin-suppressed adipogenic differentiation of MSCs}

To further investigate the involvement of PPARY in the regulation of adipogenic differentiation, PPAR $\gamma$ agonist rosiglitazone (Ros.) was added to the primary MSCs. After the rosiglitazone treatment, the PPARY and $\mathrm{aP} 2$ expression were increased by 14.8 -fold and 4.2-fold as compared to the control group. The further addition of Icaritin resulted in an increase in the inhibition of these genes expressions. The PPARY expression was suppressed by $35.4 \%, 53.8 \%$ and $71.5 \%$ in $0.1 \mu \mathrm{M}, 1 \mu \mathrm{M}$ and $10 \mu \mathrm{M}$ Icaritin groups respectively, and the aP2 expression was suppressed by $32.9 \%, 57.3 \%$ and $74.4 \%$ in $0.1 \mu \mathrm{M}, 1 \mu \mathrm{M}$ and $10 \mu \mathrm{M}$ Icaritin groups respectively. Oil-red $\mathrm{O}$ staining showed an 11.7-fold increase in adipocyte-like cells formation in rosiglitazone-treatment group as compared to the control group, and the addition of Icaritin significantly antagonized rosiglitazone-induced adipogenesis, the inhibition rate was $18.9 \%, 40.9 \%$ and $66.9 \%$ in $0.1 \mu \mathrm{M}$, $1 \mu \mathrm{M}$ and $10 \mu \mathrm{M}$ Icaritin groups respectively (Fig. 2F, $2 \mathrm{G}, 2 \mathrm{H})$.

\section{Discussion}

This study demonstrated that Icaritin promoted osteogenic differentiation of MSCs and repressed their adipogenic differentiation. We also found that the pro-osteogenic and anti-adipogenic effects of 
Icaritin were at least in part mediated by the GSK3 $\beta$ and PPARY pathways respectively. Based on these findings, we concluded that Icaritin, as the bioactive compound in herbal Epimedium-derived formula $(1,3)$, could promote osteogenic and suppress adipogenic differentiation of MSCs.

\section{Icaritin promotes osteogenic differentiation and maturation of MSCs}

Osteogenesis is a spatial cascade process, which initiates from the commitment and differentiation of bone marrow MSCs into osteoprogenitor cells and then preosteoblasts and osteoblasts, followed by matrix maturation and matrix mineralization (21). The commitment and differentiation of bone marrow MSCs towards osteoblast lineage, therefore, is the critical step in osteogenesis initiated by the activation of Runx2. The importance of this gene has been confirmed by the fact that a Runx2 gene knockout results in a complete lack of bone formation owing to maturational arrest of osteoblasts (22). During the differentiation into mature osteoblasts, a number of extracellular matrix genes are expressed by MSCs, such as bone sialoprotein, which is regarded as the middle stage marker of osteogenesis. At the late stage of bone formation, osteocalcin is up-regulated, which represents the maturation of osteoblasts and matrix mineralization processing.

As shown in the primary mice bone marrow MSCs, Icaritin up-regulated the expression of Runx2, bone sialoprotein and increased the ALP activity in a dose-dependent manner. These results suggested that Icaritin could promote the commitment and differentiation of osteoblasts in bone MSCs. In the maturation stage, the osteocalcin and matrix mineralization were also increased, suggesting that Icaritin could also promote osteoblasts maturation. In the previous published paper, Huang et al. also showed that Icaritin is more potent than herbal Epimedium-derived Icariin and icariside II in promoting osteoblastic differentiation, supporting the idea that Icaritin is a very important bioactive compound from Epimedium (23). For the underlying mechanism, as GSK3 $\beta$ could control osteogenesis through regulating Runx2 activity (24), the increased expression of Runx2 in Icaritin-treated MSCs might result from regulating GSK3 $\beta$ activity. Therefore, the GSK3 $\beta$ activity was explored in this study. The result showed that Icaritin caused phosphorylation of GSK3 $\beta$ on its Ser9 site (causing inactivation). Furthermore, the inactivation of GSK3 $\beta$ was accompanied with increased expression of $\beta$-catenin. GSK3 $\beta$ is a negative factor for controlling osteogenesis in normal situation. However, after inactivation by phosphorylation on its Ser9 site, it would activate $\beta$-catenin and initiate osteogenesis (25). These results suggested that Icaritin-induced osteogenic differentiation was associated with regulation of GSK3 $\beta$ activity.

\section{Icaritin suppresses adipogenic differentiation of MSCs}

In order to investigate whether Icaritin could inhibit adipogenic differentiation of MSCs, the adipocytes number (Oil Red $\mathrm{O}$ staining) and adipocyte-specific markers including aP2 and LPL were assessed. Adipocyte fatty acid binding protein (aP2) is known as an adipose-specific fatty acid binding protein, which is thought to be involved in intracellular transport and metabolism of fatty acids. aP2 is expressed in mature adipocyte and regarded as a terminal differentiation marker (26). Lipoprotein lipase (LPL) is thought to be an early marker of adipogenesis and highly expressed during adipogenic differentiation (27). As shown in this study, Icaritin suppressed aP2, LPL mRNA expression by inhibiting adipocyte-like cells formation. These results suggest that Icaritin could suppress the adipogenic differentiation of MSCs. For the molecular pathway, PPARY was the key transcription factor in adipocytes differentiation. In vitro study showed that forced expression of PPARY into myoblasts made their commitment into adipocytes (28). PPARY knock-down suppressed adipocyte differentiation and fat formation $(29,30)$. The present study showed that PPARY agonist rosiglitazone could induce adipocytes differentiation with enhanced expression of adipocyte-specific markers like aP2 and LPL. After Icaritin treatment, a significant suppression of adipogenic differentiation occurred. These results suggest that Icaritin inhibits adipogenesis of MSCs at least partly through PPARY -mediated pathway.

\section{Conclusion}

This was one of the first studies demonstrating that the semisynthetic molecule Icaritin could promote osteogenic differentiation and inhibit their adipogenesis of MSCs. These results demonstrate also that the pro-osteogenic effect of Icaritin is associated with regulation of GSK3 $\beta$ activity, and its anti-adipogenic effect is associated with blocking PPARY signaling. The in vitro study indicates that Icaritin has the potential for further development into anti-osteoporosis drug.

\section{Acknowledgements}

Thanks Dr. Arieh Bomzon in Consulwrite for language revision. This study was supported by Shanghai Pujiang Program (09PJ1410300), NSFC 
(30900698) and Ph.D. Programs Foundation of Ministry of Education of China (20090072120020), and Hong Kong Competitive Earmarked Research Grants (Project ID Ref. 478508 and Project ID Ref. 472508).

\section{Competing Interests}

The authors have declared that no competing interest exists.

\section{References}

1. Zhang G, Qin L, Shi Y. Epimedium-derived phytoestrogen flavonoids exert beneficial effect on preventing bone loss in late postmenopausal women: a 24-month randomized, double-blind and placebo-controlled trial. J Bone Miner Res 2007; 22(7):1072-9.

2. Zhang G, Qin L, Sheng H, Yeung KW, Yeung HY, Cheung WH, Griffith J, Chan CW, Lee KM, Leung KS. Epimedium-derived phytoestrogen exert beneficial effect on preventing steroid-associated osteonecrosis in rabbits with inhibition of both thrombosis and lipid-deposition. Bone. 2007; 40(3):685-92.

3. Zhang G, Qin L, Hung WY, Shi YY, Leung PC, Yeung HY, Leung KS. Flavonoids derived from herbal Epimedium Brevicornum Maxim prevent OVX-induced osteoporosis in rats independent of its enhancement in intestinal calcium absorption. Bone 2006; 38(6):818-25.

4. Gimble JM, Zvonic S, Floyd ZE, Kassem M, Nuttall ME. Playing with bone and fat. J Cell Biochem 2006; 98(2):251-66.

5. Griffith JF, Yeung DK, Antonio GE, Wong SY, Kwok TC, Woo J, Leung PC. Vertebral marrow fat content and diffusion and perfusion indexes in women with varying bone density: MR evaluation. Radiology 2006; 241(3):831-8

6. Astudillo P, Rios S, Pastenes L, Pino AM, Rodriguez JP. Increased adipogenesis of osteoporotic human-mesenchymal stem cells (MSCs) characterizes by impaired leptin action. J Cell Biochem 2008; 103(4):1054-65

7. Sheng H, Qin L, Zhang G, Jin WF, Wang HF, Leung KS. Colony forming unit fibroblast and their diffraction in bone marrow obtained from glucocorticoid-induced osteoporosis in rats. Osteoporosis Int. 2006; 17:322.

8. Sheng H, Zhang G, Cheung WH, Chan CW, Wang YX, Lee KM, Wang HF, Leung KS, Qin L. Elevated adipogenesis of marrow mesenchymal stem cells during early steroid-associated osteonecrosis development. J Orthop Surg 2007; 2:15.

9. Nuttall ME, Gimble JM. Controlling the balance between osteoblastogenesis and adipogenesis and the consequent therapeutic implications. Curr Opin Pharmacol 2004; 4(3):290-4.

10. Wang Z, Goh J, Das De S, Ge Z, Ouyang H, Chong JS, Low SL, Lee EH. Efficacy of bone marrow-derived stem cells in strengthening osteoporotic bone in a rabbit model. Tissue Eng. 2006; 12(7):1753-61.

11. Mukherjee S, Raje N, Schoonmaker JA, Liu JC, Hideshima T, Wein MN, Jones DC, Vallet S, Bouxsein ML, Pozzi S, Chhetri S, Seo YD, Aronson JP, Patel C, Fulciniti M, Purton LE, Glimcher LH, Lian JB, Stein G, Anderson KC, Scadden DT. Pharmacologic targeting of a stem/progenitor population in vivo is associated with enhanced bone regeneration in mice. J Clin Invest. 2008; 118(2):491-504.

12. Zhang G, Wang XL, Sheng H, Xie XH, He YX, Yao XS, Li ZR, Lee KM, He W, Leung KS, Qin L. Constitutional flavonoids derived from Epimedium dose-dependently reduce incidence of steroid-associated osteonecrosis not via direct action by themselves on potential cellular targets. PLoS One 2009; 4(7):e6419.

13. Sheng H, Qin L, Zhang G, Jin WF, Gao JJ, Wang HF, Wang YX, Tan WS. Mesenchymal Stem Cells Culture, Expansion and Their Osteogenic, Adipogenic Differentiation for Pharmaceutical Evaluations. In: Leung KS, Qin YX, Cheung WH, Qin L (eds.) A Practical Manual for Musculoskeletal Research. World Scientific, New York, 2008::39-62.

14. Tropel P, Noel D, Platet N, Legrand P, Benabid AL, Berger F. Isolation and characterisation of mesenchymal stem cells from adult mouse bone marrow. Exp Cell Res. 2004; 295(2):395-406.

15. Tam KF, Cheung WH, Lee KM, Qin L, Leung KS. Delayed stimulatory effect of low-intensity shockwaves on human periosteal cells. Clin Orthop Relat Res. 2005; 438:260-5.

16. Rucci N, Rufo A, Alamanou M, Teti A. Modeled microgravity stimulates osteoclastogenesis and bone resorption by increasing osteoblast RANKL/OPG ratio. J Cell Biochem. 2007; 100(2):464-73.

17. Dang ZC, van Bezooijen RL, Karperien M, Papapoulos SE, Lowik CW. Exposure of KS483 cells to estrogen enhances osteogenesis and inhibits adipogenesis. J Bone Miner Res. 2002; 17(3):394-405.
18. Huang L, Teng XY, Cheng YY, Lee KM, Kumta SM. Expression of preosteoblast markers and Cbfa-1 and Osterix gene transcripts in stromal tumour cells of giant cell tumour of bone. Bone 2004; 34(3):393-401.

19. Chen X, McClurg A, Zhou GQ, McCaigue M, Armstrong MA, Li G. Chondrogenic differentiation alters the immunosuppressive property of bone marrow-derived mesenchymal stem cells, and the effect is partially due to the upregulated expression of B7 molecules. Stem Cells. 2007; 25(2):364-70.

20. Zhang G, Sheng H, He YX, Xie XH, Wang $Y X$, Lee KM, Yeung KW, Li ZR, He W, Griffith JF, Leung KS, Qin L. Continuous occurrence of both insufficient neovascularization and elevated vascular permeability in rabbit proximal femur during inadequate repair of steroid-associated osteonecrotic lesions. Arthritis Rheum 2009; 60(10):2966-77.

21. Long MW. Osteogenesis and bone-marrow-derived cells. Blood Cells Mol Dis. 2001; 27(3):677-90.

22. Komori T, Yagi H, Nomura S, Yamaguchi A, Sasaki K, Deguchi K, Shimizu Y, Bronson RT, Gao YH, Inada M, Sato M, Okamoto R, Kitamura Y, Yoshiki S, Kishimoto T. Targeted disruption of Cbfa1 results in a complete lack of bone formation owing to maturational arrest of osteoblasts. Cell 1997; 89(5):755-64.

23. Huang J, Yuan L, Wang X, Zhang TL, Wang K. Icaritin and its glycosides enhance osteoblastic, but suppress osteoclastic, differentiation and activity in vitro. Life Sci. 2007; 81(10):832-40.

24. Kugimiya F, Kawaguchi H, Ohba S, Kawamura N, Hirata M, Chikuda H, Azuma Y, Woodgett JR, Nakamura K, Chung UI. GSK-3beta controls osteogenesis through regulating Runx2 activity. PLoS One 2007; 2(9):e837.

25. Baron R, Rawadi G. Wnt signaling and the regulation of bone mass. Curr Osteoporos Rep. 2007; 5(2):73-80.

26. Shaughnessy S, Smith ER, Kodukula S, Storch J, Fried SK. Adipocyte metabolism in adipocyte fatty acid binding protein knockout mice (aP2-/-) after short-term high-fat feeding: functional compensation by the keratinocyte [correction of keritinocyte] fatty acid binding protein. Diabetes 2000; 49(6):904-11.

27. Garcia E, Lacasa M, Agli B, Giudicelli Y, Lacasa D. Modulation of rat preadipocyte adipose conversion by androgenic status: involvement of C/EBPs transcription factors. J Endocrinol. 1999; 161(1):89-97.

28. Yamanouchi K, Ban A, Shibata S, Hosoyama T, Murakami Y, Nishihara M. Both PPARgamma and C/EBPalpha are sufficient to induce transdifferentiation of goat fetal myoblasts into adipocytes. J Reprod Dev. 2007; 53(3):563-72.

29. Hosono T, Mizuguchi H, Katayama K, Koizumi N, Kawabata K, Yamaguchi T, Nakagawa S, Watanabe Y, Mayumi T, Hayakawa T. RNA interference of PPARgamma using fiber-modified adenovirus vector efficiently suppresses preadipocyte-to-adipocyte differentiation in 3T3-L1 cells. Gene 2005; 348:157-65.

30. Rosen ED, Sarraf P, Troy AE, Bradwin G, Moore K, Milstone DS, Spiegelman BM, Mortensen RM. PPAR gamma is required for the differentiation of adipose tissue in vivo and in vitro. Mol Cell 1999; 4(4):611-7. 